\title{
Quasi-static Torque Profile Expressions for Magnetic Resonance Based Remote Actuation
}

\author{
Matthias Vandeputte ${ }^{1,2}$, Luc Dupré ${ }^{1}$ and Guillaume Crevecoeur ${ }^{1,2}$
}

\begin{abstract}
Resonant wireless power transfer has been employed to transfer electrical power from a transmitter to a receiver coil over an air gap and to remotely charge consumer devices. In this paper, the receiver coil is replaced by a stator-rotor topology, enabling magnetic resonance based motoring over substantial air gaps. The principles of resonant wireless power transfer are used to induce currents in a strongly coupled magnetic resonance system. This results in its turn in torque, which is applied on the rotor body, allowing for remote actuation. We propose a voltage or current controlled magnetic resonance motoring topology, for which we derive expressions for the generated torque depending on the rotor angle. Furthermore, torque profile expressions are derived for motoring systems with multiple stator and/or rotor coils. Finally, an experimental setup is built to validate the obtained torque expressions. Using the validated expressions, we present a sensitivity analysis of the key system parameters with respect to the torque profile. The presented torque profile expressions enable further topology exploration and optimization of magnetic resonance based motoring systems.
\end{abstract}

Index Terms-Coils, electromagnetic forces, magnetic resonance, mutual coupling, RLC circuits

\section{INTRODUCTION}

$\mathbf{T}$ HE field of wireless power transfer (WPT) [1]-[5], also known as inductively coupled power transfer or ICPT [6], [7], recently attracted a rapidly growing research interest. In a strongly coupled magnetic resonance system (SCMR), electric energy is transferred between two (or more) strongly magnetically coupled resonator coils. Such an inductor-capacitor (RLC) series connection has a certain resonance frequency and by matching the resonance frequency of both the transmitter coil (Tx) and receiver coil(s) (Rx), it is possible to efficiently and selectively [8], [9] transfer power over significant air gaps. WPT has found applications from low power systems, e.g. the charging of consumer electronics [10], [11], as well as medium power [12] to high power developments, such as charging an electric vehicle when parked over an inductive charging pad [13], [14].

Remote actuation, i.e. having actuation at a certain location without having physical power cables connected to the actuator, is possible by means of an SCMR system. Figure 1 shows three system topologies for SCMR based remote actuation. Most often, the receiver coil of the SCMR system and the actuator (motor $\mathrm{M}$ ) sides are indirectly connected through an energy buffer, e.g. a battery (topology a). A more direct energy conversion methodology (b) is to rectify the induced voltage in

\footnotetext{
${ }^{1}$ M. Vandeputte, L. Dupré and G. Crevecoeur are with the Department of Electrical Energy, Metals, Mechanical Constructions \& Systems, Ghent University, 9000 Ghent, Belgium matthias .vandeputte@UGent .be.

${ }^{2} \mathrm{M}$. Vandeputte and G. Crevecoeur are with the EEDT-DC, Flanders Make.
}

an SCMR receiver coil and then use this DC voltage directly to actuate, for example, a DC-motor [12], [15], [16].

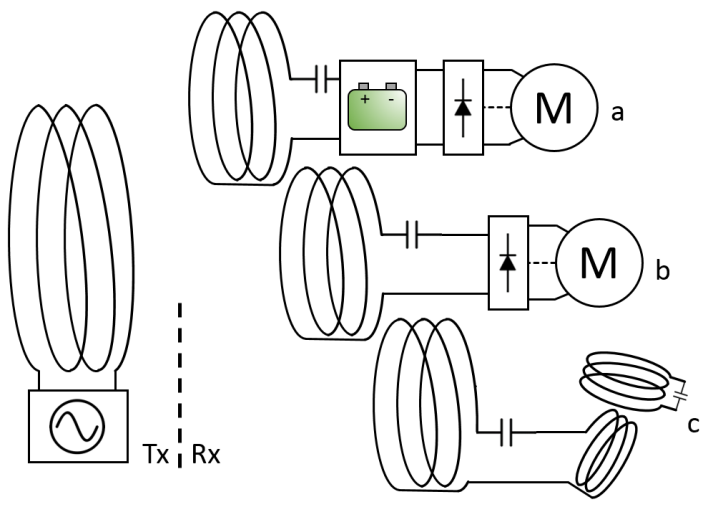

Figure 1: The excited current in the receiver coil can be used for remote actuation of motor $\mathrm{M}$ by (a) storing the transferred power in an energy buffer, e.g. a battery, (b) rectifying the current and sending it to a standard actuator or (c) by applying the excited current(s) directly to induce forces and torques.

Thus, this paper explores an even more direct approach to using SCMR for remote actuation (c). We allow one or more resonator coils in the SCMR system to rotate around an axis. Further, the currents induced in the resonator coils by an external magnetic field can be used directly to effectuate forces or torques on an actuator body, such as a plunger or a rotor axle [17]. The latter topology eliminates the requirement of power electronic conversion at the receiver side. Additionally, the torque can be controlled from the transmitter side by controlling the current or voltage amplitude.

The excitation frequency of the transmitter in an SCMR motoring system is much larger than (and thus independent of) the mechanical rotating frequency. As such, we can assume quasi-static induction of currents in the SCMR coils when deriving torque profiles of the topology depicted in Figure 1(c). The above mechanism is contrary to classical rotating machines, such as an induction motor or a synchronous motor, where the excitation frequency of the current in the stator winding is close or equal to the rotating frequency times the number of pole pairs.

In order to achieve, optimize and design remote actuation based on SCMR, it is paramount to gain insight on how the specifications of the resonator coils and their coupling affect their ability to generate torque. Therefore, in this paper, we propose closed form expressions for the quasi-static torque generated by a certain system of strongly coupled magnetic 
resonator coils. These expressions are derived starting from the electromechanical power flows within the SCMR system. We consider general resonator topologies having an arbitrary number of transmitter coils, fixed stator coils and rotor coils, which are attached to the actuated body (i.e. a linear actuator or a rotor body). Subsequently, the torque profile expressions are experimentally validated for a system consisting of one transmitter, one stator coil and one rotor coil. The effect of adding a second rotor coil is also investigated using the derived expressions. Finally, the effect of the resonator coils' quality and the coupling between the resonator coils on the torque generation are assessed using the validated quasi-static torque profiles. We anticipate that more thorough designs on the level of the individual resonators, as well as the full system topology, will become feasible using the presented expressions.

In Section II-A, the topology of an SCMR motoring system is explained and matrix formulations for the system currents for both a voltage and current controlled transmitter power source are derived. The power flows in the SCMR system are quantified and closed form expressions for the quasi-static torque profile are derived in Section II-B. Section III describes the validation of the previously derived torque profile using an experimental setup. Section IV provides a discussion of the key parameters in the SCMR motoring system and the effect of adding a second rotor coil on the torque generation.

\section{QUASI-STATIC SCMR COIL INTERACTION}

An SCMR motoring system of topology (c) in Figure 1 consists of an arbitrary number of three distinct circuit types, which are shown in Figure 2, namely the transmitter coil $(t)$, stator coils $(s)$ and rotor coils $(r)$. The transmitter coil is connected to a voltage or current controlled power source. The stator coils and rotor coils are inductor-capacitor (RLC) series connections, of which the stator coils are fixed in space and the rotor coils are free to rotate around an axis. As well, the rotation angle of the rotor is denoted as $\theta$.

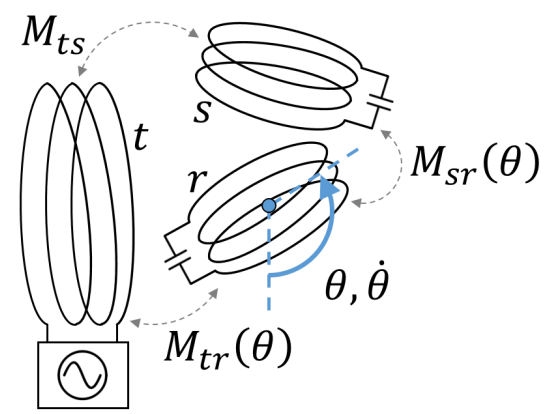

Figure 2: The general resonator topology consists of a transmitter coil $(t)$ connected to a power source, a stator coil $(s)$ that is fixed in space and one or more rotor coils $(r)$ that can rotate around an axis.

\section{A. State-space model}

When a current is present in a coil, a voltage is induced in another coil when these coils are magnetically coupled. The magnetic interaction can be described by Faraday's law of induction, which states that the induced electromotive force (EMF) $\varepsilon_{a b}$ in a conducting loop $a$ by current $i_{b}$ is equal to the negative of the rate of change of the enclosed magnetic flux $n \Phi_{a b}$. Here $n$ is the amount of turns in a coil and $\Phi_{a b}$ is the flux excited by current $i_{b}$ enclosed in one turn of coil $a$. Since coil $b$ can rotate for topology (c) in Figure 1, the EMF consists of the sum of the effects of movement (angle $\theta$ ) and change in excitation current $i_{b}$, and can be derived by applying the chain rule:

$$
\begin{aligned}
\varepsilon_{a b} & =-n \frac{\mathrm{d} \Phi_{a b}}{\mathrm{~d} t} \\
& =\frac{\mathrm{d}}{\mathrm{d} t}\left(M_{a b}(\theta) \cdot i_{b}\right) \\
& =\frac{\mathrm{d} M_{a b}(\theta)}{\mathrm{d} \theta} \frac{\mathrm{d} \theta}{\mathrm{d} t} i_{b}+M_{a b}(\theta) \frac{\mathrm{d} i_{b}}{\mathrm{~d} t} \\
& =K_{a b}(\theta) \dot{\theta} i_{b}+M_{a b}(\theta) \frac{\mathrm{d} i_{b}}{\mathrm{~d} t}
\end{aligned}
$$

The first part of Equation (1) is called motional EMF generated by the Lorentz force and the second part is the transformer EMF induced by the changing magnetic field. $M_{a b}$ represents the mutual inductance between circuits $a$ and $b$. The spatial derivative of $M_{a b}(\theta)$ to the rotation angle $\theta$ is denoted as $K_{a b}(\theta)$ and the rotational speed as $\dot{\theta}$. Note that the reciprocal mutual inductance values $M_{a b}$ and $M_{b a}$ are equal and the same holds for the spatial derivative, namely $K_{a b}$ and $K_{b a}$. Based on (1), we can construct a state-space representation of the electrical states in the SCMR system. For each coil, we express Kirchoff's voltage law (KVL):

$$
v_{a}=R_{a} i_{a}+L_{a} \frac{\mathrm{d}}{\mathrm{d} t} i_{a}+v_{C_{a}}+\sum_{b \neq a} \varepsilon_{a b}
$$

with $v_{a}$ as the voltage from an external power source $(=0$ if no source is present), $R_{a}$ as the equivalent series resistance (ESR), $L_{a}$ as the coil self inductance and $v_{C_{a}}$ as the voltage over the capacitor $C_{a}$ (if a series-capacitor is present). For each capacitor, the associated differential equation is added:

$$
C_{a} \frac{\mathrm{d}}{\mathrm{d} t} v_{C_{a}}=i_{a}
$$

In order to convert these equations to matrix form, we define resistance matrix $R_{d}$, impedance matrix $Z_{d}$, voltage vector $V_{t}$ and state vector $x$ as follows:

$$
R_{d}=\left[\begin{array}{ccccc}
R_{t} & 0 & 0 & 0 & 0 \\
0 & R_{s} & 0 & 0 & 0 \\
0 & 0 & R_{r} & 0 & 0 \\
0 & 0 & 0 & 0 & 0 \\
0 & 0 & 0 & 0 & 0
\end{array}\right]
$$

$$
Z_{d}=\left[\begin{array}{ccccc}
L_{t} & M_{t s} & M_{t r}(\theta) & 0 & 0 \\
M_{t s}^{T} & L_{s} & M_{s r}(\theta) & 0 & 0 \\
M_{t r}(\theta)^{T} & M_{s r}(\theta)^{T} & L_{r} & 0 & 0 \\
0 & 0 & 0 & C_{s} & 0 \\
0 & 0 & 0 & 0 & C_{r}
\end{array}\right]
$$




$$
V_{t}=\left[\begin{array}{c}
v_{t} \\
0 \\
0 \\
0 \\
0
\end{array}\right] ; x=\left[\begin{array}{c}
i_{t} \\
i_{s} \\
i_{r} \\
v_{C s} \\
v_{C r}
\end{array}\right]
$$

Here, $v_{t}$ is the voltage over the transmitter coil and $x$ is the vector of all electrical states, namely, the currents in the coils and the voltage across the series-capacitors. If there are multiple $\left(n_{r}\right)$ rotor coils present in the system, $M_{s r}(\theta)$ is an $1 \times n_{r}$ vector. Equations (2) and (3) can now be written in matrix form:

$$
V_{t}=Z_{d} \dot{x}+\left(R_{d}+\dot{Z}_{d}\right) x
$$

\section{B. General torque expression}

In order to derive the general expression for the torque, we examine the energy transfer per unit of time $(\dot{E})$ in the system of coils. The energy $E$ that is stored in the capacitors and the magnetic field can be expressed as:

$$
E=\frac{1}{2} x^{T} Z_{d} x
$$

Application of the chain rule gives:

$$
\dot{E}=\frac{1}{2} \dot{x}^{T} Z_{d} x+\frac{1}{2} x \dot{Z}_{d} x+\frac{1}{2} x^{T} Z_{d} \dot{x}
$$

Equation (7) can be rewritten as:

$$
Z_{d} \dot{x}=-\left(R_{d}+\dot{Z}_{d}\right) x+V_{t}
$$

such that Equation (9) simplifies to:

$$
\dot{E}=V_{t}^{T} x-x^{T}\left(R_{d}+\frac{1}{2} \dot{Z}_{d}\right) x
$$

We can distinguish two components in Equation (11): the input power $\left(P_{i n}=v_{t} i_{t}=V_{t}^{T} x\right)$ and the dissipated power $\left(P_{\text {diss }}=R_{t} i_{t}^{2}+R_{s} i_{s}^{2}+R_{r} i_{r}^{2}=x^{T} R_{d} x\right)$. Mechanical work is the remaining term that relates to $\dot{E}$ due to the conservation of energy:

$$
\dot{E}=P_{i n}-P_{\text {diss }}-T \dot{\theta}
$$

and therefore:

$$
T \dot{\theta}=\frac{1}{2} x^{T} \dot{Z}_{d} x
$$

As $Z_{d}$ is a function of the position $\theta$, the time derivative of $Z_{d}$ can be rewritten as:

$$
T \dot{\theta}=\frac{1}{2} x^{T} \frac{\partial\left[Z_{d}(\theta(t))\right]}{\partial t} x=\frac{1}{2} x^{T} \dot{\theta} \frac{\partial\left[Z_{d}(\theta)\right]}{\partial \theta} x
$$

Elimination of $\dot{\theta}$ in both sides of (14) results in the general expression for the torque:

$$
T(t)=\frac{1}{2} x(t)^{T} \frac{\partial\left[Z_{d}(\theta)\right]}{\partial \theta} x(t)
$$

The torque is now shown to be independent of the speed $\dot{\theta}$. For the limit case of $\dot{\theta}=0$, (15) also holds. The average torque over one cycle is then equal to:

$$
T_{a v}(\theta)=\frac{1}{4} X^{*} \frac{\partial\left[Z_{d}(\theta)\right]}{\partial \theta} X
$$

with $X=\left[\begin{array}{lllll}\hat{\mathrm{i}}_{t} & \hat{\mathrm{i}}_{s} & \hat{\mathrm{i}}_{r} & \hat{\mathrm{v}}_{C s} & \hat{\mathrm{v}}_{C r}\end{array}\right]^{T}$ the phasor representation of the states $(x) . X^{*}$ is the conjugate transpose of $X$. Only the mutual inductance elements of $Z_{d}$ are variable in $\theta$, such that the capacitor voltages do not directly contribute to the torque. The average torque per cycle can now be written as:

$$
T_{a v}(\theta)=\frac{1}{4}\left(I^{*}\left[\begin{array}{ccc}
0 & 0 & K_{t r}(\theta) \\
0 & 0 & K_{s r}(\theta) \\
K_{t r}(\theta)^{T} & K_{s r}(\theta)^{T} & 0
\end{array}\right] I\right)
$$

with $I=\left[\begin{array}{lll}\hat{\mathrm{i}}_{t} & \hat{\mathrm{i}}_{s} & \hat{\mathrm{i}}_{r}\end{array}\right]^{T}$ the vector of the complex phasors of $i_{t}, i_{s}$ and $i_{r} . K_{a b}$ was defined in Equation (1) as the angular derivative of $M_{a b}$.

\section{Voltage and current controlled transmitters}

A voltage or current controlled transmitter gives rise to magnetically coupled coils whose electrical state $x$ in (6), together with the voltage over the transmitter coil $V_{t}$, follow dynamics (7). When considering a voltage controlled transmitter, $V_{t}$ is a constant imposed value, whereas in case of a current controlled transmitter, $V_{t}$ implicitly depends on the imposed current $i_{t}$ and satisfies the electrical circuit laws in the transmitter coil. When deriving the general torque expression in Section II-B, we defined $I$ as the vector of complex current phasors in the transmitter, stator and rotor coils. We will now express the current vector $I$ as a function of the input, namely phasor $\hat{\mathrm{v}}_{t}$ for a voltage controller transmitter and $\hat{\mathrm{i}}_{t}$ for a current controlled transmitter. Let us recall that in the frequency domain, the induced voltage expressed in (1) can be split up in an in-phase part with respect to the excitation coil current phasor $\hat{\mathrm{i}}_{b}$ (motional EMF) and an out-of-phase part (transformer EMF):

$$
\hat{\varepsilon}_{a b}=K_{a b}(\theta) \dot{\theta}_{\hat{\mathrm{i}}_{b}}+j \omega M_{a b}(\theta) \hat{\mathrm{i}}_{b}
$$

with $\omega$ as the electrical angular speed, and $\omega=2 \pi f$ and $f$ as the frequency of the power source. For the calculation of the quasi-static torque, we assume that $\dot{\theta} \ll \omega$, such that the element $K_{a b} \dot{\theta}$ can be neglected in the calculation of the current vector $I$ without significant loss in accuracy. This is equivalent to removing the $\dot{Z}_{d} x$ term in (7), such that:

$$
V_{t} \approx Z_{d} \dot{x}+R_{d} x
$$

1) Voltage controlled transmitter power source: When applying the KVL in the frequency domain, only one complex equation is required per coil. For a voltage controlled transmitter, Equation (19) turns into a set of three complex algebraic equations that connect the electrical states of the voltage controlled SCMR system:

$$
Z_{V}\left[\begin{array}{c}
\hat{\mathrm{i}}_{t} \\
\hat{\mathrm{i}}_{s} \\
\hat{\mathrm{i}}_{r}
\end{array}\right]=\left[\begin{array}{c}
\hat{\mathrm{v}}_{t} \\
0 \\
0
\end{array}\right] \Leftrightarrow\left[\begin{array}{c}
\hat{\mathrm{i}}_{t} \\
\hat{\mathrm{i}}_{s} \\
\hat{\mathrm{i}}_{r}
\end{array}\right]=Z_{V}^{-1}\left[\begin{array}{l}
1 \\
0 \\
0
\end{array}\right] \hat{\mathrm{v}}_{t}
$$

with impedance matrix $Z_{V}$ :

$$
\begin{aligned}
& Z_{V}= \\
& {\left[\begin{array}{ccc}
R_{t}+j \omega L_{t} & j \omega M_{t s} & j \omega M_{t r}(\theta) \\
j \omega M_{t s}^{T} & R_{s}+\frac{1}{j \omega C_{s}}+j \omega L_{s} & j \omega M_{s r}(\theta) \\
j \omega M_{t r}(\theta)^{T} & j \omega M_{s r}(\theta)^{T} & R_{r}+\frac{1}{j \omega C_{r}}+j \omega L_{r}
\end{array}\right]}
\end{aligned}
$$


It is important to note that the torque (17) scales quadratically with the amplitude of the transmitter voltage. We consider an SCMR system with one coil of each type, i.e. one transmitter, stator and rotor coil. In what follows, this topology will be referred to as the single rotor SCMR motoring system. For the considered system, all matrix elements of (21) have a $1 \times 1$ size, as there is only one transmitter, stator and rotor coil present in the system. In (20), the current vector $I$ is expressed as a function of the transmitter current $\hat{\mathrm{v}}_{t}$ and impedance matrix $Z_{V}$. Note that all stator and rotor coils are assumed to be tuned to resonant conditions $\left(j \omega L_{a}+\frac{1}{j \omega C_{a}}=0\right)$. Inserting the current vector from (20) in torque expression (17) gives the following simplified expression:

$$
\begin{aligned}
& \quad T_{1}(\theta)= \\
& \frac{1}{2} \frac{M_{t s} \omega^{2}\left(K_{s r} M_{t r}-K_{t r} M_{s r}\right)\left(\omega^{2} M_{s r}^{2}+R_{r} R_{s}\right)}{\left(\begin{array}{c}
{\left[R_{t}\left(\omega^{2} M_{s r}^{2}+R_{r} R_{s}\right)+\omega^{2} M_{t r}^{2} R_{s}+\omega^{2} M_{t s}^{2} R_{r}\right]^{2}} \\
+\left[\omega L_{t}\left(\omega^{2} M_{s r}^{2}+R_{r} R_{s}\right)-2 \omega^{3} M_{s r} M_{t r} M_{t s}\right]^{2}
\end{array}\right)} \mathrm{v}_{t}^{2}
\end{aligned}
$$

2) Current controlled transmitter power source: In the case of a controlled current in the transmitter coil, the applied voltage is an indirect result of the imposed current $\hat{\mathrm{i}}_{t}$, such that the order of the system decreases by one:

$$
\begin{aligned}
\hat{\mathrm{v}}_{t} & =\left(R_{t}+j \omega L_{t}\right) \hat{\mathrm{i}}_{t}+Z_{t}^{T}\left[\begin{array}{l}
\hat{\mathrm{i}}_{s} \\
\hat{\mathrm{i}}_{r}
\end{array}\right] \\
0 & =Z_{I}\left[\begin{array}{l}
\hat{\mathrm{i}}_{s} \\
\hat{\mathrm{i}}_{r}
\end{array}\right]+Z_{t} \hat{\mathrm{i}}_{t} \Leftrightarrow\left[\begin{array}{l}
\hat{\mathrm{i}}_{s} \\
\hat{\mathrm{i}}_{r}
\end{array}\right]=-Z_{I}^{-1} Z_{t} \hat{\mathrm{i}}_{t}
\end{aligned}
$$

with impedance matrix $Z_{I}$ and $Z_{t}$ :

$$
\begin{aligned}
& Z_{I}=\left[\begin{array}{cc}
R_{s}+\frac{1}{j \omega C_{s}}+j \omega L_{s} & j \omega M_{s r}(\theta) \\
j \omega M_{s r}(\theta)^{T} & R_{r}+\frac{1}{j \omega C_{r}}+j \omega L_{r}
\end{array}\right] \\
& Z_{t}=\left[\begin{array}{ll}
j \omega M_{t s} & j \omega M_{t r}(\theta)
\end{array}\right]^{T}
\end{aligned}
$$

As with the voltage controlled transmitter power source, here we consider a single rotor SCMR motoring system. The torque expression (22) in case of having a voltage controlled transmitter scales quadratically with $\mathrm{v}_{t}$; correspondingly, we can deduce from (17) together with (23) that the torque scales quadratically with the amplitude of the transmitter current $i_{t}$ in the case of a current controlled transmitter. This results in the following simplified torque expression:

$$
T_{1}(\theta)=\frac{1}{2} \frac{M_{t s} \omega^{2}\left(K_{s r} M_{t r}-K_{t r} M_{s r}\right)}{\omega^{2} M_{s r}^{2}+R_{r} R_{s}} \mathrm{i}_{t}^{2}
$$

with $\mathrm{i}_{t}$ the amplitude of current $i_{t}$.

Torque profile expressions (22) and (25) were derived for the case of a single rotor SCMR motoring system. Appendix A details the torque expression for a system consisting of multiple rotors for both a voltage controlled (35) and current controlled transmitter (36).

\section{EXPERIMENTAL VALIDATION OF THE TORQUE EXPRESSIONS}

In the previous section, we derived closed form torque expressions for a single rotor SCMR system, specifically for both a voltage (22) and a current controlled (25) transmitter. An experimental SCMR setup is constructed to validate the derived torque expressions and to demonstrate the motoring possibilities based on magnetic resonance.

Figure 3(a) schematically presents the experimental setup and in (b) we depict the actual experimental SCMR setup. We have a transmitter coil $(t)$ with about 200 turns of $0.8 \mathrm{~mm}$ that are wound on a curved rectangle, having a height of $8 \mathrm{~cm}$ and a depth of $8 \mathrm{~cm}$. This transmitter coil can be voltage or current controlled. The stator coil $(s)$ is series connected to a capacitor and is short circuited, while the geometrical properties of the stator coil are similar to the transmitter coil. Next to the transmitter and stator coil, we consider a rotor coil, consisting of $0.8 \mathrm{~mm}$ wire wound with 200 turns, having a diameter of about $5 \mathrm{~cm}$ and a depth of $8 \mathrm{~cm}$. In this experimental setup, a single rotor (coil $r_{1}$ ) and a dual rotor (coils $r_{1}$ and $r_{2}$ ) can be considered. $r_{1}$ and $r_{2}$ have similar geometrical and magnetic properties and are placed perpendicular to each other. This second rotor coil is added in an attempt to smoothen the torque profile of a single rotor SCMR motoring system. As well, the inside of the rotor is filled with toroidal ferrite cores to improve the magnetic coupling between the system coils. Further, the air gaps between transmitter and rotor are large, around 1 $\mathrm{cm}$. For driving frequencies around the resonance frequency of both the rotor and stator coils, the induced currents reach adequate values that result in motion of the rotor.

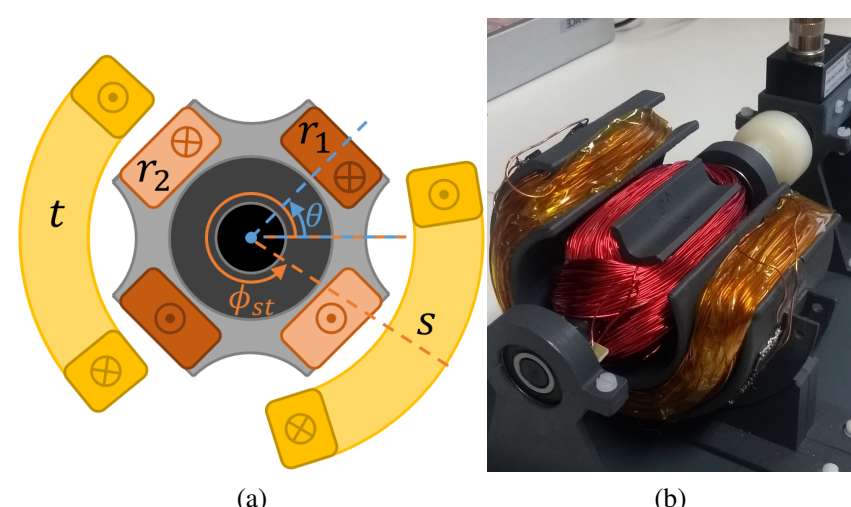

Figure 3: The experimental setup (schematic in (a) and actual in (b)) to validate the derived torque expressions and to demonstrate motoring possibilities of SCMR. The setup consists of a transmitter and stator that have a relatively large air gap with the rotor. The experimental setup consists of a single coil rotor $\left(r_{1}\right)$ and there is the possibility of having a second rotor coil $\left(r_{2}\right)$. Ferrite magnetic material (dark grey material in (a)) is inserted in the rotor.

A resonance frequency of around $6530 \mathrm{~Hz}$ is elected to optimize the quality factor or Q-factor $\left(Q_{a}\right)$ of the coils.

$$
Q_{a}=\frac{2 \pi f L_{a}}{R_{a}(f)}
$$


The ESR of the stator $\left(R_{s}\right)$ and rotor $\left(R_{r}\right)$ coils are measured for resonant conditions, together with the series resistance $\left(R_{t}\right)$ and inductance $\left(L_{t}\right)$ of the transmitter coil and the mutual inductance between the transmitter and stator $\left(M_{t s}\right)$. These values are listed in Table I.

Table I: Transmitter, rotor and stator coil values of the experimental setup depicted in Figure 3 were measured at resonance $(6530 \mathrm{~Hz})$.

\begin{tabular}{c|c}
\hline Parameter & Value \\
\hline \hline$L_{t}$ & $5.45 \mathrm{mH}$ \\
$R_{t}$ & $4.47 \Omega$ \\
$R_{r}$ & $4.50 \Omega$ \\
$R_{s}$ & $4.55 \Omega$ \\
$M_{t s}$ & $0.84 \mathrm{mH}$ \\
\hline
\end{tabular}

All signal acquisitions are done using the DSpace 1104 platform. The exerted torque is measured using a Lorenz Messtechnik DR-2112 torque transducer with a nominal torque of $1 \mathrm{Nm}( \pm 0.1 \%)$, which also acts as an incremental encoder. The friction torque in the bearings is estimated around $3 \mathrm{Nmm}$ by measuring the torque while rotating the rotor slowly in both directions.

\section{A. Single rotor SCMR motoring system}

The experimental setup with single rotor coil is used for validating torque expression (22). We use the parameter values listed in Table I and measured the mutual inductance profiles, as well as their spatial derivative. Figure 4 shows the measured $M_{t r}$ and $K_{t r}$ (a) $M_{s r}$ and $K_{s r}$ (b) profiles for the single rotor coil. These values are obtained by measuring the excitation current of one coil and the induced voltage in the other.

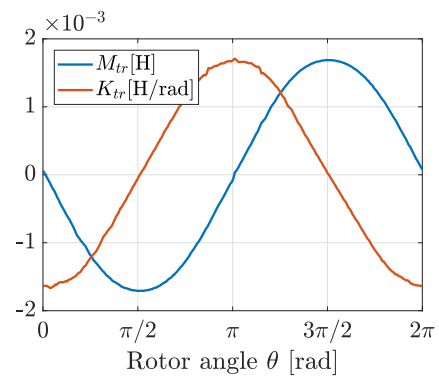

(a)

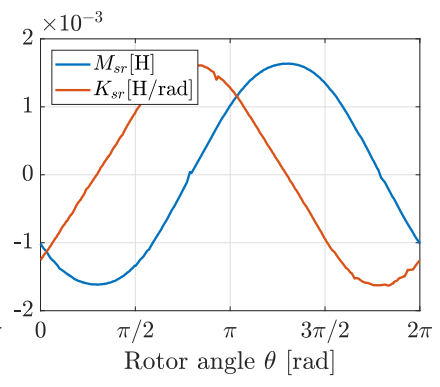

(b)
Figure 4: Measured transmitter-rotor (a) and stator-rotor (b) mutual inductance values $\left(M_{t r}\right.$ and $\left.M_{s r}\right)$ and their respective spatial derivatives $\left(K_{t r}\right.$ and $\left.K_{s r}\right)$.

One can observe that the mutual inductance profile resembles a sinusoidal function of the rotor angle. Figure $5 \mathrm{com}-$ pares the simulated torque profile using (22) with the actual rotor torque measurement when having a voltage controlled transmitter voltage $\left(v_{t}\right)$ with RMS amplitude of $150 \mathrm{~V}$. As the friction torque is around $3 \mathrm{Nmm}$ in our experimental setup, we conclude that the peak torque can be predicted accurately, both in position and magnitude, when compared to experimental measurements. This concludes the validation of the single rotor SCMR motoring system.

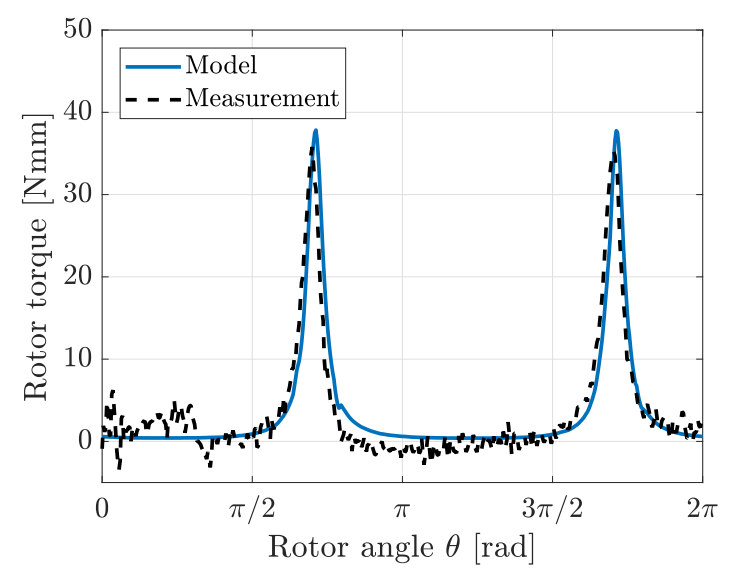

Figure 5: Measured and simulated rotor torque as a function of rotor angle $\theta$ in a single rotor SCMR system with voltage controlled transmitter coil. $v_{t}$ has a RMS amplitude of $150 \mathrm{~V}$.

\section{B. Dual rotor SCMR motoring system}

The experimental setup with two rotor coils $\left(r_{1}\right.$ and $r_{2}$ coils in Figure 3 ) is employed for validating the torque expression derived in (35) with $n_{r}=2$. Furthermore, we investigate whether adding a second rotor coil perpendicular to the first rotor coil can smoothen the torque profile compared to the highly variable torque profile of the single rotor SCMR from Figure 5. Inserting the system parameters in expression (35) results in an expected torque of only $0.1 \mathrm{Nmm}$. This torque is far below the friction torque of $3 \mathrm{Nmm}$. Correspondingly, no movement was experimentally observed. Moreover, we measured the currents in the system coils to validate the electrical part of the model. The measured amplitude and phase for these currents are compared against their expected values in Figure 6. The reason for having no movement is because the stator is always strongly coupled with at least one of both rotor resonators, such that the stator can never reach resonant behavior. As a consequence, the rotor current is out of phase with the stator current for most rotor positions. This can be observed in the measured current phase in Figure 6 (b). The phase of the rotor current only crosses that of the stator current when the rotor current amplitude is at its minimal value, i.e. here around $\frac{3 \pi}{4}$ and $\frac{7 \pi}{4}$. Both influences eliminate the possibility of effective torque generation.

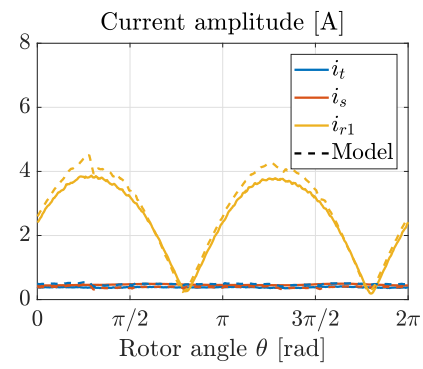

(a)

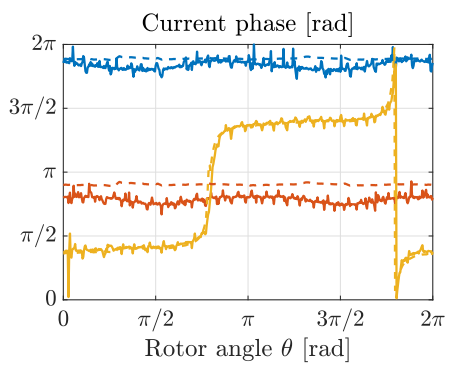

(b)
Figure 6: The measured currents in the dual rotor system match the expected system currents both in amplitude (a) and phase (b). Only one of both rotor currents is shown for clarity, as they only differ by a spatial shift of $\frac{\pi}{2}$. 


\section{Discussion}

Starting from the validated torque profile expressions from previous Section III, we discuss the effect of important system parameters on the motoring capabilities of SCMR in case of having a voltage (Section IV-A) or current (IV-B) controlled single rotor SCMR motoring system and in case of a dual rotor SCMR motoring (IV-C) system.

\section{A. Voltage controlled single rotor SCMR motoring system}

When analyzing the torque profile expression (22) in case of a voltage controlled transmitter, it is apparent that the torque is strongly affected by the Q-factor of the coil and the coupling factor $k$ (or the mutual inductance $M_{a b}$ ) between the coils. Figure 7 depicts the effect of the Q-factor on the torque profile by multiplying the quality factors of the resonators $\left(Q_{s}\right.$ and $Q_{r}$ ) by a so-called Q-factor multiplier. A multiplier of one corresponds to the experimental setup. Thus, the average torque of the simulated setup is used as a reference for normalization and corresponds with the red line in Figure 7.

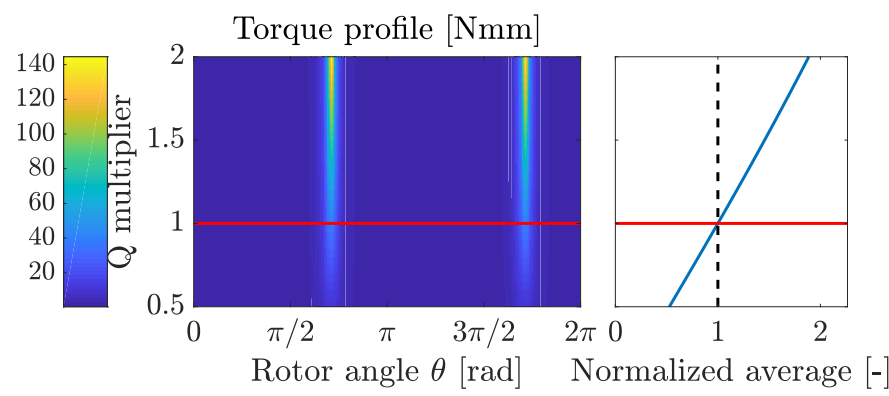

Figure 7: The peak torque in the torque profile (left) and the average normalized torque (right) increase together with the quality factor of both the stator and the rotor coil.

Figure 7 shows that the peak torque values increase together with the Q-factors. This is because the induced currents in the resonators are higher for increasing Q-factors. Also, the peak torque remains located around the same angle $\theta$. Figure 8 shows the torque profile for varying coupling factors, by rescaling the coupling factors of the simulated system $\left(k_{t r}\right.$, $k_{s r}$ and $k_{t s}$ ) by a so-called $k$ multiplier.

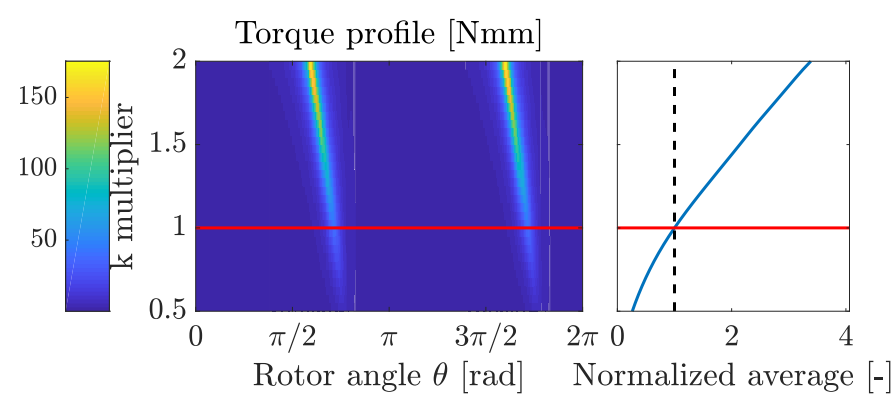

Figure 8: The peak torque in the torque profile (left) and the average normalized torque (right) increase together with the coupling factors between all coils. A skew of the torque profile can be observed such that the peak torque occurs for different angles depending on the coupling.
Similarly to the effect of the changing Q-factors, the torque increases for the whole profile when increasing the coupling factors in the system. However, a skew in the peak torque position can be observed. When having higher coupling between the coils, peak torques thus occur for positions corresponding to non-zero coupling between stator and rotor coils.

\section{B. Current controlled single rotor SCMR motoring system}

The torque profile of a current controlled system (25) can be studied qualitatively by assuming sinusoidal mutual inductance profiles, as experimentally observed in Figure 4. For this, we only consider the first spatial harmonic of $M_{t r}$ and $M_{s r}$. The angular dependence of the mutual inductances and associated spatial derivatives can be described as:

$$
\begin{aligned}
M_{t r_{1}}(\theta) & =-\mathbb{M}_{t r} \sin (\theta) \\
M_{s r_{1}}(\theta) & =-\mathbb{M}_{s r} \sin \left(\theta-\phi_{s t}\right) \\
K_{t r_{1}}(\theta) & =-\mathbb{K}_{t r} \cos (\theta)=-\mathbb{M}_{t r} \cos (\theta) \\
K_{s r_{1}}(\theta) & =-\mathbb{K}_{s r} \cos \left(\theta-\phi_{s t}\right)=-\mathbb{M}_{s r} \cos \left(\theta-\phi_{s t}\right)
\end{aligned}
$$

with $\mathbb{M}_{t r}$ and $\mathbb{M}_{s r}$ the amplitude of $M_{t r_{1}}$ and $M_{s r_{1}}$ and $\phi_{s t}$ the physical angle shift between them (also indicated in Figure 3(a)). Substituting terms (27) in (25) gives rise to the following torque expression for a current controlled transmitter:

$$
\begin{aligned}
T_{1}(\theta) & =\frac{1}{2} \frac{M_{t s} \omega^{2}\left[\begin{array}{c}
\mathbb{M}_{s r} \cos \left(\theta-\phi_{s t}\right) \mathbb{M}_{t r} \sin (\theta) \\
-\mathbb{M}_{t r} \cos (\theta) \mathbb{M}_{s r} \sin \left(\theta-\phi_{s t}\right)
\end{array}\right]}{\omega^{2}\left[\mathbb{M}_{s r} \sin \left(\theta-\phi_{s t}\right)\right]^{2}+R_{r} R_{s}} \\
& =\frac{1}{2} \frac{\omega^{2} M_{t s} \mathbb{M}_{t r} \mathbb{M}_{s r} \sin \left(\phi_{s t}\right)}{\omega^{2} \mathbb{M}_{s r}^{2} \sin ^{2}\left(\theta-\phi_{s t}\right)+R_{r} R_{s}} \mathrm{i}_{t}^{2}
\end{aligned}
$$

From (28) it is clear that the torque is unidirectional if the mutual inductance profiles can be approximated by their first spatial harmonic. The motoring direction is determined by the angle $\phi_{s t}$. In what follows, a quantification of the peak torque, the minimum torque and the average torque is made for the torque profile described in (28). As such, the peak torque can easily be found for $\sin \left(\theta-\phi_{s t}\right)=0$, which corresponds to the rotor not being magnetically coupled with the stator:

$$
T_{1, \text { peak }}=\frac{1}{2} \frac{\omega^{2} M_{t s} \mathbb{M}_{t r} \mathbb{M}_{s r} \sin \left(\phi_{s t}\right)}{R_{r} R_{s}} \mathrm{i}_{t}^{2}
$$

We can deduce from (29) that the maximum torque increases for increasing levels of coupling between the coils whereas an increase in coil resistance (inversely proportional to the Q-factor) degrades the torque.

The minimum torque is found for $\sin \left(\theta-\phi_{s t}\right)= \pm 1$ :

$$
T_{1, \min }=\frac{1}{2} \frac{\omega^{2} M_{t s} \mathbb{M}_{t r} \mathbb{M}_{s r} \sin \left(\phi_{s t}\right)}{\omega^{2} \mathbb{M}_{s r}^{2}+R_{r} R_{s}} \mathrm{i}_{t}^{2}
$$

The minimum torque is an important indication for the self-starting possibilities of the topology. Ideally, the minimum torque should exceed the friction torque. The condition $\sin \left(\theta-\phi_{s t}\right)= \pm 1$ is satisfied when the rotor and stator coils are aligned. In other words, magnetic interaction between the stator and rotor results in a degradation of the torque generation. 
The average torque over one cycle can be found as:

$$
\begin{aligned}
T_{1, \text { av }} & =\frac{\sin \left(\phi_{s t}\right) \omega^{2} M_{t s} \mathbb{M}_{t r} \mathbb{M}_{s r} \mathrm{i}_{t}^{2}}{4 \pi \omega^{2} \mathbb{M}_{s r}^{2}} \int_{0}^{2 \pi} \frac{\mathrm{d} \theta}{\sin ^{2}\left(\phi_{s t}-\theta\right)+\frac{R_{r} R_{s}}{\omega^{2} \mathbb{M}_{s r}^{2}}} \\
& =\frac{\sin \left(\phi_{s t}\right) \omega^{2} M_{t s} \mathbb{M}_{t r} \mathbb{M}_{s r} \mathrm{i}_{t}^{2}}{2 \omega^{2} \mathbb{M}_{s r}^{2}} \frac{1}{\sqrt{\frac{R_{r} R_{s}}{\omega^{2} \mathbb{M}_{s r}^{2}}\left(1+\frac{R_{r} R_{s}}{\omega^{2} \mathbb{M}_{s r}^{2}}\right)}} \\
& \triangleq \frac{\sin \left(\phi_{s t}\right) M_{t s} \mathbb{M}_{t r} \mathrm{i}_{t}^{2}}{2 \mathbb{M}_{s r}} \frac{\alpha_{s r, \max }^{2}}{\sqrt{\alpha_{s r, \max }^{2}+1}}
\end{aligned}
$$

with

$$
\alpha_{s r, \max }^{2}=\frac{\omega^{2} \mathbb{M}_{s r}^{2}}{R_{r} R_{s}}=\frac{\omega^{2}\left(k_{s r, \max } \sqrt{L_{r} L_{s}}\right)^{2}}{\frac{\omega^{2} L_{r} L_{s}}{Q_{r} Q_{s}}}=k_{s r, \max }^{2} Q_{r} Q_{s}
$$

The product of the coupling factor and the Q-factor of resonator coils $(\alpha=k Q)$ is a common figure of merit for the efficiency of WPT systems [18], [19].

\section{Dual rotor SCMR motoring system}

No significant torque was generated in our dual rotor SCMR motoring setup. We analyze, in this subsection, the dual rotor SCMR in more detail. Similar to Section IV-B, the torque profile can be approximated by considering only the first spatial harmonic of $M_{t r_{2}}$ and $M_{s r_{2}}$. The elements of (33) considering the added rotor coil are shifted over an angle of $\frac{\pi}{2}$ compared to (27):

$$
\begin{aligned}
M_{t r_{2}}(\theta) & =-\mathbb{M}_{t r} \cos (\theta) \\
M_{s r_{2}}(\theta) & =-\mathbb{M}_{s r} \cos \left(\theta-\phi_{s t}\right) \\
K_{t r_{2}}(\theta) & =\mathbb{M}_{t r} \sin (\theta) \\
K_{s r_{2}}(\theta) & =\mathbb{M}_{s r} \sin \left(\theta-\phi_{s t}\right)
\end{aligned}
$$

When filling in these terms in (37), the following expression results:

$$
\begin{aligned}
T_{2}(\theta) & =\frac{1}{2} \frac{\omega^{2} M_{t s} \mathbb{M}_{t r} \mathbb{M}_{s r}\left[\sin \left(\phi_{s t}\right)+\sin \left(\phi_{s t}\right)\right]}{\omega^{2} \mathbb{M}_{s r}^{2}\left[\cos ^{2}\left(\theta-\phi_{s t}\right)+\sin ^{2}\left(\theta-\phi_{s t}\right)\right]+R_{r} R_{s}} \mathrm{i}_{t}^{2} \\
& =\frac{\sin \left(\phi_{s t}\right) \omega^{2} M_{t s} \mathbb{M}_{t r} \mathbb{M}_{s r}}{\omega^{2} \mathbb{M}_{s r}^{2}+R_{r} R_{s}} \mathrm{i}_{t}^{2}
\end{aligned}
$$

One can deduce from (34) that since the denominator for the torque is constant, the torque for the two rotor system is also constant when assuming sinusoidal mutual inductance profiles. Also, the direction of the torque depends on the sign of $\sin \left(\phi_{s t}\right)$. Therefore, the torque reaches a maximum absolute value for $\sin \left(\phi_{s t}\right)= \pm 1$. For this condition, a rotor coil is aligned with the transmitter coil when there is no coupling with the stator coil. However, there is a trade-off in torque, as the coupling between the transmitter and stator $M_{t s}$ is also affected by the angle $\phi_{s t}$.

We can conclude that the added rotor coil is capable of removing the torque ripple, but the magnetic interaction between the stator and at least one of the rotor coils at all times severely degrades the maximum achievable torque. The possibly high peak torque is eliminated, while the (now constant) torque of the two rotor system is only double the minimum torque of the single rotor system.

Appendix B describes a qualitative way to compare the single and dual rotor topologies considered in this paper (40): only for $\alpha_{s r \text {, max }}^{2}$ values under 3 , the dual rotor system is considered to have the higher average quasi-static torque. For the setup used, $\alpha_{s r, \max }^{2}$ was around 273 and thus, significantly larger than 3. From this condition, one can conclude that in the search for the highest coil quality factors and magnetic coupling between system coils (which in turn, increases the value of $\alpha_{s r, \max }^{2}=k_{s r, \max }^{2} Q_{s} Q_{r}$ value of the system), the single rotor topology will be more effective.

\section{CONCLUSION}

In this paper, principles of wireless power transfer are used to induce currents in a strongly coupled magnetic resonance system, ultimately resulting in remote actuation over large air gaps. Quasi-static torque profile expressions for remote actuation are drawn based on quasi-static SCMR coil interactions. Generic torque expressions in closed form are presented, as well as specific torque profiles when having voltage or current controlled transmitters. We specifically considered topologies that consist of a single rotor coil or a dual rotor coil. These expressions were subsequently validated using an experimental SCMR motoring setup. We demonstrated the possibility of remote actuation in case of single rotor motoring system and discussed the reason of having no significant torque in the dual rotor system. Finally, the effect of key system parameters being the Q-factor of the resonator coils and the coupling between the system coils on the torque profile (both peak torque as average torque) is analyzed. The peak torque scales with the Qfactor, whereas a skew in torque profile can be observed when increasing the coupling factors. We demonstrated, based on both simulations and measurements, that the average torque of the single rotor coil system is significantly higher than for the dual rotor coil system. The considered experimental setup has a relatively low (average) torque. This can be attributed to the relatively large air gap of approximately $1 \mathrm{~cm}$ and the limited amount of magnetic material that was inserted inside the rotor body. We demonstrated that resonant coupling enables remote actuation in this non optimized system. The authors are convinced that future development of SCMR motoring systems by exploring various topologies and optimizations has become tractable using the derived expressions for the quasistatic torque profile.

\section{APPENDIX A}

\section{CLOSED FORM TORQUE EXPRESSIONS FOR MULTIPLE RESONATOR COILS}

Equation (17) can also be worked out into a closed form expression for the dual rotor coil system. We assume that all rotor coils have the same resistance $\left(R_{r_{j}}=R_{r}\right)$ and that the mutual inductance between the rotor coils is zero or negligible. For a voltage controlled dual rotor SCMR system, the torque profile can be expressed as follows: 


$$
\begin{gathered}
T_{2}(\theta)= \\
\frac{\frac{1}{2} M_{t s} \omega^{2} \sum_{i=1}^{2}\left(K_{s r_{i}} M_{t r_{i}}-K_{t r_{i}} M_{s r_{i}}\right)\left(\omega^{2} \sum_{i=1}^{2} M_{s r_{i}}+R_{r} R_{s}\right) \mathrm{v}_{t}^{2}}{\left(\left[\omega^{2} \sum_{i=1}^{2} M_{t r_{i}}^{2} R_{s}+\omega^{2} M_{t s}^{2} R_{r}+\omega^{4} \frac{\left(M_{s r_{1}} M_{t r_{2}}-M_{s r_{2}} M_{t r_{1}}\right)^{2}}{R_{r}}\right]^{2}\right)} \\
+\left[\omega L_{t}\left(\omega^{2} \sum_{i=1}^{2} M_{s r_{i}}^{2}+R_{r} R_{s}\right)-2 \omega^{3} M_{t s} \sum_{i=1}^{2}\left(M_{s r_{i}} M_{t r_{i}}\right)\right]^{2}
\end{gathered}
$$

For a current controlled transmitter and multiple $\left(n_{r}\right)$ rotor coils, (36) is obtained when neglecting the mutual induction between the rotor coils.

$$
T_{n_{r}}(\theta)=\frac{1}{2} \frac{M_{t s} \omega^{2} \sum_{i=1}^{n_{r}}\left[\left(K_{s r_{i}} M_{t r_{i}}-K_{t r_{i}} M_{s r_{i}}\right) \prod_{i \neq j} R_{r_{j}}\right]}{\omega^{2} \sum_{i=1}^{n_{r}}\left[M_{s r_{i}}^{2} \prod_{i \neq j} R_{r_{j}}\right]+R_{s} \prod_{j} R_{r_{j}}} \mathrm{i}_{t}^{2}
$$

If all rotor resistance values are assumed equal, (36) simplifies to:

$$
T_{n_{r}}(\theta)=\frac{1}{2} \frac{M_{t s} \omega^{2} \sum_{i=1}^{n_{r}}\left(K_{s r_{i}} M_{t r_{i}}-K_{t r_{i}} M_{s r_{i}}\right)}{\omega^{2} \sum_{i=1}^{n_{r}} M_{s r_{i}}^{2}+R_{r} R_{s}} \mathrm{i}_{t}^{2}
$$

\section{APPENDIX B \\ COMPARISON OF AVERAGE TORQUE FOR SINGLE AND DUAL ROTOR SCMR MOTORING SYSTEMS}

In Section IV-B and IV-C, the torque profiles and their average values were derived for the single and dual rotor SCMR motoring systems respectively. If the ratio of these average torques (38) is larger than unity, the dual rotor setup would be beneficial not only for the elimination of the torque ripple, but also from an efficiency standpoint.

$$
\begin{aligned}
\frac{T_{2, \mathrm{av}}}{T_{1, \mathrm{av}}} & =\frac{2 \sqrt{R_{r} R_{s}} \sqrt{\omega^{2} \mathbb{M}_{s r}^{2}+R_{r} R_{s}}}{\omega^{2} \mathbb{M}_{s r}^{2}+R_{r} R_{s}} \\
& =\frac{2 \sqrt{R_{r} R_{s}}}{\sqrt{\omega^{2} \mathbb{M}_{s r}^{2}+R_{r} R_{s}}}
\end{aligned}
$$

The ratio $\frac{T_{2, \text { av }}}{T_{1, \text { av }}}$ will be larger than unity for:

$$
\frac{1}{3} \omega^{2} \mathbb{M}_{s r}^{2}<R_{r} R_{s}
$$

or in terms of the dimensionless system parameter in the single rotor coil (32):

$$
\alpha_{s r, \max }^{2}<3
$$

\section{ACKNOWLEDGMENT}

Matthias Vandeputte holds a doctoral grant strategic basic research of the Fund for Scientific Research Flanders (FWO). The authors furthermore acknowledge the FWO (project G0D9316N) and the BOF (project 01N02716).

\section{REFERENCES}

[1] Andre Kurs, Aristeidis Karalis, Robert Moffatt, John D Joannopoulos, Peter Fisher, and Marin Soljačić. Wireless power transfer via strongly coupled magnetic resonances. science, 317(5834):83-86, 2007.

[2] Alanson P Sample, David T Meyer, and Joshua R Smith. Analysis, experimental results, and range adaptation of magnetically coupled resonators for wireless power transfer. IEEE Transactions on Industrial Electronics, 58(2):544-554, 2011.

[3] Shu Yuen Ron Hui, Wenxing Zhong, and Chi Kwan Lee. A critical review of recent progress in mid-range wireless power transfer. IEEE Transactions on Power Electronics, 29(9):4500-4511, 2014.

[4] Olutola Jonah and Stavros V Georgakopoulos. Wireless power transfer in concrete via strongly coupled magnetic resonance. IEEE Transactions on Antennas and Propagation, 61(3):1378-1384, 2013.

[5] Xun Liu, WM Ng, CK Lee, and SY Hui. Optimal operation of contactless transformers with resonance in secondary circuits. In Applied Power Electronics Conference and Exposition, 2008. APEC 2008. Twenty-Third Annual IEEE, pages 645-650. IEEE, 2008.

[6] Grant A Covic and John T Boys. Inductive power transfer. Proceedings of the IEEE, 101(6):1276-1289, 2013.

[7] JT Boys, GA Covic, and A Williamson Green. Stability and control of inductively coupled power transfer systems. IEE Proceedings-Electric Power Applications, 147(1):37-43, 2000.

[8] Dukju Ahn and Songcheol Hong. Effect of coupling between multiple transmitters or multiple receivers on wireless power transfer. IEEE Transactions on Industrial Electronics, 60(7):2602-2613, 2013.

[9] Yiming Zhang, Ting Lu, Zhengming Zhao, Fanbo He, Kainan Chen, and Liqiang Yuan. Selective wireless power transfer to multiple loads using receivers of different resonant frequencies. IEEE Transactions on Power Electronics, 30(11):6001-6005, 2015.

[10] Chang-Gyun Kim, Dong-Hyun Seo, Jung-Sik You, Jong-Hu Park, and Bo-Hyung Cho. Design of a contactless battery charger for cellular phone. IEEE Transactions on Industrial Electronics, 48(6):1238-1247, 2001.

[11] SY Hui. Planar wireless charging technology for portable electronic products and qi. Proceedings of the IEEE, 101(6):1290-1301, 2013.

[12] Ali Abdolkhani, Aiguo Patrick Hu, and Nirmal-Kumar C Nair. A double stator through-hole type contactless slipring for rotary wireless power transfer applications. IEEE Transactions on Energy Conversion, 29(2):426-434, 2014.

[13] Siqi Li and Chunting Chris Mi. Wireless power transfer for electric vehicle applications. IEEE journal of emerging and selected topics in power electronics, 3(1):4-17, 2015.

[14] Jaegue Shin, Seungyong Shin, Yangsu Kim, Seungyoung Ahn, Seokhwan Lee, Guho Jung, Seong-Jeub Jeon, and Dong-Ho Cho. Design and implementation of shaped magnetic-resonance-based wireless power transfer system for roadway-powered moving electric vehicles. IEEE Transactions on Industrial Electronics, 61(3):1179-1192, 2014.

[15] Chaoqiang Jiang, KT Chau, TW Ching, Chunhua Liu, and Wei Han. Time-division multiplexing wireless power transfer for separately excited dc motor drives. IEEE Transactions on Magnetics, 53(11):1-5, 2017.

[16] Konstantinos D Papastergiou and D Ewen Macpherson. An airborne radar power supply with contactless transfer of energy - part i: Rotating transformer. IEEE Transactions on Industrial Electronics, 54(5):28742884, 2007.

[17] M Boyvat, C Hafner, and J Leuthold. Wireless control and selection of forces and torques-towards wireless engines. Scientific reports, 4:5681, 2014.

[18] Tatsuki Ujihara, Quang-Thang Duong, and Minoru Okada. kq-product analysis of inductive power transfer system with two transmitters and two receivers. In Wireless Power Transfer Conference (WPTC), 2017 IEEE, pages 1-4. IEEE, 2017.

[19] Takashi Ohira. Extended kq product formulas for capacitive-and inductive-coupling wireless power transfer schemes. IEICE Electronics Express, 11(9):20140147-20140147, 2014. 


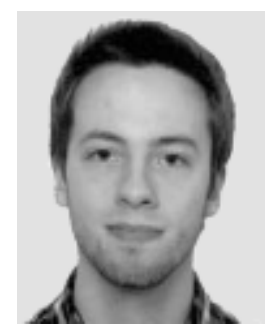

Matthias Vandeputte was born in Ghent, Belgium, in 1993. In 2016, he received the M.Sc. degree in electrical and mechanical engineering from Ghent University, Ghent, Belgium.

In 2015, he interned at the Simulation and Modeling department of ArcelorMittal, Ghent, as a Systems Engineer. He joined the Department of Electrical Energy, Metals, Mechanical Constructions and Systems at Ghent University as Ph.D. after graduating, in 2016. Since 2018, he is a SB fellow of the Research Foundation - Flanders. His current research interests are semi-analytical and numerical methods in electromagnetics, sizing and topology optimization, applied on interacting resonant structures.

$\mathrm{He}$ is an affiliate member of Flanders Make, the strategic research centre for the manufacturing industry in Flanders, Belgium.

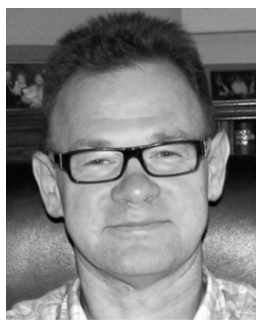

Luc Dupré was born in 1966. He received the electrical and mechanical engineering degree and the $\mathrm{Ph} . \mathrm{D}$. degree in applied sciences from the Ghent University, Belgium, in 1989 and 1995, respectively. In 1989, he joined the Department of Electrical Energy, Systems and Automation, Ghent University, as a research assistant. In 1996, he was a postdoctoral researcher for the Fund of Scientific Research-Flanders and in 1998 for six months a visiting post-doctoral fellow at Istituto Elettrotecnico Nazionale 'Galileo Ferraris', in Torino (Italy). In 2002, he became an associate professor at the Faculty of Engineering and Architecture, Ghent University and since 2006 he is full professor at that faculty. His research interests mainly include numerical methods for low frequency electromagnetism (in particular for electrical machines), modeling and characterization of soft magnetic materials, micromagnetism, inverse problems and optimization in (bio)electromagnetism.

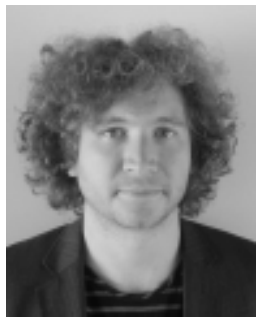

Guillaume Crevecoeur $\left({ }^{\circ}\right.$ 1981) received the MSc and the $\mathrm{PhD}$ degree in Engineering Physics from Ghent University in 2004 and 2009, respectively. In 2004 he joined the Department of Electrical Energy, Metals, Mechanical Constructions, and Systems as doctoral student and in 2009 he became a postdoctoral fellow of the Research Foundation Flanders (FWO-Flanders). In the winter 2011 he was a visiting researcher at the Technical University Ilmenau and the Physikalische Technische Bundesanstalt, Berlin, Germany. In 2014 he was appointed Associate Professor at the Faculty of Engineering and Architecture of Ghent University. He is furthermore active member of Flanders Make, the strategic research center for the manufacturing industry, where he leads the Ghent University core lab on Sensing, Monitoring, Decision-making and Control. His research interests are the modelling, optimization and control of dynamical systems including foundational work on model-based optimization algorithms, inverse problems and nonlinear optimal control with strategic research on electromechanical and biomedical applications. 